\title{
Correlations of low-field NMR and variable-field NMR parameters with osteoarthritis in human articular cartilage under load
}

\author{
Erik Rössler ${ }^{1}$ | Carlos Mattea ${ }^{1}$ | Simo Saarakkala ${ }^{2,3}$ | Petri Lehenkari ${ }^{2}$ | Mikko Finnilä ${ }^{2,3}$ | \\ Lassi Rieppo $^{2,3}$ | Sakari Karhula ${ }^{2,3}$ | Miika T. Nieminen ${ }^{2,3,4}$ | Siegfried Stapf ${ }^{1}$ (i)
}

${ }^{1}$ Department of Technical Physics II, TU

Ilmenau, Ilmenau, Germany

${ }^{2}$ Research Unit of Medical Imaging, Physics and Technology, University of Oulu, P.O. Box 5000, Oulu, Finland

${ }^{3}$ Medical Research Center, University of Oulu and Oulu University Hospital, P.O. Box 50, Oulu, Finland

${ }^{4}$ Department of Diagnostic Radiology, Oulu University Hospital, P.O. Box 50, Oulu, Finland

Correspondence

S. Stapf, Department of Technical Physics II,

TU Ilmenau, IImenau, Germany.

Email: siegfried.stapf@tu-ilmenau.de

Funding information

EU Horizon 2020 collaborative project

IDentIFY, Grant/Award Number: 668119
NMR experiments carried out at magnetic fields below $1 \mathrm{~T}$ provide new relaxation parameters unavailable with conventional clinical scanners. Contrast of $T_{1}$ generally becomes larger towards low fields, as slow molecular reorientation processes dominate relaxation at the corresponding Larmor frequencies. This advantage has to be considered in the context of lower sensitivity and frequently reduced spatial resolution. The layered structure of cartilage is one example where a particularly strong variation of $T_{1}$ across the tissue occurs, being affected by degenerative diseases such as osteoarthritis (OA). Furthermore, the presence of ${ }^{1} \mathrm{H}-{ }^{14} \mathrm{~N}$ cross-relaxation, leading to so-called quadrupolar dips in the ${ }^{1} \mathrm{H}$ relaxation time dispersion, provide insight into the concentration and mobility of proteoglycans and collagen in cartilage, both being affected by OA. In this study, low-field imaging and variable-field NMR relaxometry were combined for the first time for tissue samples, employing unidirectional load to probe the mechanical properties. 20 human knee cartilage samples were placed in a compression cell, and studied by determining relaxation profiles without and with applied pressure $(0.6 \mathrm{MPa})$ at $50 \mu \mathrm{m}$ in-plane resolution, and comparing with volume-averaged $T_{1}$ dispersion. Samples were subsequently stored in formalin, prepared for histology and graded according to the Mankin score system.

Quadrupolar dips and thickness change under load showed the strongest correlation with Mankin grade. Average $T_{1}$ and change of maximum $T_{1}$ under load, as well as its position, correlate with thickness and thickness change. Furthermore, $T_{1}(\omega)$ above $25 \mathrm{mT}$ was found to correlate with thickness change. While volume-averaged $T_{1}$ is not a suitable indicator for $\mathrm{OA}$, its change due to mechanical load and its extreme values are suggested as biomarkers available in low-field MRI systems. The shape of the dispersion $T_{1}(\omega)$ represents a promising access to understanding and quantifying molecular dynamics in tissue, pointing toward future in vivo tissue studies.

\section{KEYWORDS}

cartilage, low-field NMR, mechanical load, osteoarthritis, quadrupolar dips, relaxometry

\section{1 | INTRODUCTION}

Clinical MRI investigation of cartilage tissue in joints and spine has seen growing importance in the recent decade and has become a standard and routine procedure for a number of health issues; however, the main approach of clinical studies rests on topological information, such as distances between bone surfaces and peripheral tissue such as ligaments and tendons. Common to the tissue of interest is the comparatively short transverse relaxation time, which limits the use of conventional imaging sequences and potentially compromises the theoretical spatial resolution. This leads to the fact that cartilage tissue, with its typical thickness of $2-3 \mathrm{~mm}$, is resolved only into a few pixels at most. Nevertheless, a wealth of information 
has been derived from clinical ${ }^{1}$ and ex vivo studies, in particular concerning one of the most common diseases, osteoarthritis (OA). Among other quantities, the value of $T_{2}$ and its orientational dependence, ${ }^{2-5}$ the analysis of $T_{1 \rho},{ }^{6-8}$ the diffusion coefficient and its anisotropy ${ }^{2,9}$ and the change of these parameters under mechanical load were investigated, ${ }^{10-12}$ and correlations with the severity of the disease were established. A recent book gives a summary of the state of the art of MRI studies on cartilage. ${ }^{13}$ The vast majority of these studies were carried out either at typical clinical field strength of 1.5-3 T, or on dedicated high-field scanners with even stronger magnetic field gradients and much higher spatial resolution.

In recent years, a new generation of low-field solutions, either whole-body or extremity scanners, have entered the market; they combine reduction in cost with higher flexibility, for instance by allowing tilting of the patient together with the detection system in order to compare the state in joints with and without load, ${ }^{14}$ using the effect of the actual body weight. Alternatively, this has been achieved with mechanical devices designed for compressing, for example, the lower extremities, ${ }^{15}$ and has been demonstrated to be successfully applicable for the determination of the patellofemoral joint cartilage contact area at $0.5 \mathrm{~T} .{ }^{16,17}$ While low magnetic field strengths inevitably lead to a loss of signal-to-noise ratio, and consequentially of spatial resolution, measurements at lower fields often experience higher contrast and potentially hold extra information not available at typical clinical field strengths. In an early work, the superiority of $T_{1}$ versus $T_{2}$ contrast in cartilage imaging at $0.15 \mathrm{~T}$ was already pointed out; ${ }^{18}$ dedicated devices for extremities have been presented, ${ }^{19}$ and routines for OA prediction at $0.18 \mathrm{~T}$ discussed. ${ }^{20}$ While the diagnostic potential of low-field MRI is questioned, the failure of high-field studies in early-stage diagnostics of OA stated and the importance of relaxation studies for the latter identified in Reference 21, Reference 22 confirms that early-stage and follow-up studies can successfully be carried out with low-field scanners.

Improved contrast is mainly expected to be based on $T_{1}$, which is known to show stronger variation in a wide range of non-biological complex fluid and solid systems towards lower fields, ${ }^{23}$ and the existence of cross-relaxation phenomena between ${ }^{1} \mathrm{H}$ and ${ }^{14} \mathrm{~N}$ of nitrogen-containing compounds such as proteins and collagen. ${ }^{24}$ In the context of medical imaging, 'low fields' can loosely be considered as field strengths below about $0.5 \mathrm{~T}$. However, studies in this area have been rather limited to this date. In Reference 25 it was shown that the variation of ${ }^{1} \mathrm{H} \mathrm{T}_{1}$ over the cross-section of mammalian cartilage can amount to a factor of 3-5 at a field of $0.27 \mathrm{~T}$, similar to if not larger than the variation in $T_{2}$ at any field strength. By comparison, $T_{1}$ at high field varies significantly less; in a study carried out at $9.4 \mathrm{~T}$, a correlation of $T_{1}$ with water content in bovine cartilage could be established. ${ }^{26}$ However, water relaxation depends not only on water content but also on the kind of interaction with existing interfaces. $T_{1}$ is more robust to measure and is independent of orientation; like $T_{2}$, it allows, when measured at low fields, the distinction of the three zones of cartilage (superficial, transition, radial), which are all affected by disease. This information is available with dedicated lowfield scanners of high spatial resolution, such as the NMR-MOUSE (Mobile Universal Surface Explorer ${ }^{\mathrm{TM}}$; Magritek, Aachen, Germany) providing resolution mainly along one dimension, ${ }^{27}$ which however is suitable for materials with layered structure such as cartilage. Measurement of crossrelaxation, the so-called quadrupolar dips in relaxation, on the other hand, require hardware with variable magnetic field strength in the region of these features, i.e. 50-80 mT. So far, only one group has presented spatially resolved data within this field regime, ${ }^{28-30}$ with resolutions insufficient to separate the details within cartilage tissue. An alternative approach suggests the use of an additional field coil that is switched on for a defined period of time before the actual imaging pulse sequence. With this design, the frequency dependence of $T_{1}$ is determined by obtaining relaxation parameter images at two similar, but different field strengths; the difference in $T_{1}$ is then approximated by a gradient $\mathrm{d} T_{1} / \mathrm{d} B_{0}$ and employed for tissue characterization. ${ }^{31,32}$ To date, however, volume-averaged studies on field-cycling relaxometers remain the standard tool for investigating frequency dependences in tissue. ${ }^{23}$

In this contribution, the aim is first to quantify the presence or absence of correlations between a number of low-field and variable-field parameters that are accessible with two different commercial hardware units, and second to identify such parameters that are related to OA and can be suitable for low-field MRI studies with a limited spatial resolution. To this end, a total of 20 human knee cartilage samples were studied and graded according to the Mankin score systems in order to allow a quantitative comparison with the state of OA.

\section{2 | METHODS}

A total of 20 osteochondral plug samples of $6 \mathrm{~mm}$ diameter were extracted from human tibial plateaus from patients undergoing total knee arthroplasty, and were stored frozen at $-20^{\circ} \mathrm{C}$ in tubes filled with phosphate-buffered saline (PBS). ${ }^{33}$ The osteochondral samples were from nine patients (age range 66-89; four females and five males). The plugs were taken from different, non-weight-bearing locations of the tibial plateau where tissue appeared visually healthy or degenerated, respectively. The experiments were approved by the Ethical Committee of the Northern Ostrobothnia Hospital District, Oulu, Finland (191/2000).

Each sample was allowed to thaw in PBS and equilibrate for $24 \mathrm{~h}$ at $+6^{\circ} \mathrm{C}$ in order to minimize the possible effect of degradation, before being exposed to room temperature, and was then placed in a tightly fitting cylindrical container that allowed the application of mechanical load in the vertical direction via a hydrostatic pressure cell, with small holes drilled in order to release excess water. This cell was mounted on top of an NMRMOUSE single-sided scanner (Magritek) operating at a ${ }^{1} \mathrm{H}$ Larmor frequency of $11.7 \mathrm{MHz}$ and possessing a constant vertical magnetic field gradient of $11.5 \mathrm{~T} / \mathrm{m}$, and the relaxation times $T_{2}$ and $T_{1}$ of the tissue were determined with a one-dimensional resolution of $50 \mu \mathrm{m}$, averaging over the cylinder diameter of $6 \mathrm{~mm}$. While $\mathrm{T}_{2}$ was measured using a Carr-Purcell-Meiboom-Gill (CPMG) train with a $180^{\circ}$ pulse length of $3.5 \mu$ s and a pulse separation of $88 \mu \mathrm{s}, T_{1}$ experiments were carried out with a saturation recovery sequence and signal acquired by a CPMG train in order to enhance signal-to-noise ratio; signal intensity was obtained by integrating over the first six echoes of the CPMG sequence, corresponding to a total time of 
$550 \mu \mathrm{s}$, in order to ensure that $T_{2}$ decay during this period was negligible. All decays were fitted by monoexponential functions. The thickness of the cartilage was defined between the surface $(0 \mu \mathrm{m})$ and the onset of constant $T_{1}$ in the calcified zone. The same experiment was repeated under constant vertical pressure of $0.6 \mathrm{MPa}$ afterwards, following a 20 min equilibration period. Note that, for practical reasons, a resolution of 50 um was considered fully sufficient and feasible with respect to typical layer thickness and total experimental duration, which was of the order of 5$6 \mathrm{~h}$, while the actual limit of the hardware has been determined to be $20 \mu \mathrm{m}$ for aqueous systems. ${ }^{34}$ From these experiments, the following parameters were derived: maximum values of $T_{1}$ and $T_{2}$, respectively, within the tissue with/without pressure, and their percentile difference; position of the same, in micrometers from the surface and as percentage relative to the thickness; ratio of maximum and minimum relaxation times without pressure; peak signal intensity with/without pressure and percentile difference; ratio of integrated signal with/without pressure; thickness with/ without pressure and percentile difference.

Samples were then taken out of the cell and the cartilage was separated from the bone and calcified tissue. The cartilage samples were subsequently measured in a SpinMaster 2000 Fast Field Cycling relaxometer (Stelar, Mede, Italy), and the $T_{1}$ dispersion was obtained in the frequency range $10 \mathrm{kHz}-20 \mathrm{MHz}$ with particular emphasis on the region of quadrupolar dips between $1.5 \mathrm{and} 4 \mathrm{MHz}$, where sampling took place with higher density. The area under the quadrupolar peaks, expressed as relaxation rate $R_{1}=1 / T_{1}$, was determined by subtracting the background dispersion by means of a polynomial best fit. All signals were acquired at a detection field corresponding to a Larmor frequency of $16.7 \mathrm{MHz}$, following a single $90^{\circ}$ pulse and integrating the FID. The signal decays were fitted to an exponential function; no significant deviation from monoexponential behavior was observed within the accuracy of these experiments. All NMR experiments were carried out at room temperature. From these experiments, the following parameters were derived: power-law exponent for frequencies below and above $1 \mathrm{MHz}$, respectively; area of the quadrupolar peaks in terms of $R_{1}$; average $T_{1}$ at the same field as with NMR-MOUSE, corresponding to $11.7 \mathrm{MHz} ; T_{1}$ at lowest accessed field, corresponding to $10 \mathrm{kHz}$.

Following the NMR protocol, samples were stored in a $10 \%$ formalin solution, then cut, stained and categorized according to the Mankin ${ }^{35}$ and Osteoarthritis Research Society International ${ }^{36}$ grading system by three individuals, the results of which were averaged. It has been shown before that the two grading systems show a strong correlation; ${ }^{37}$ we find similarly high correlation coefficients between the two grades, 0.95 . It is thus justified to present only one of them, and we restrict our discussion to the Mankin grade. Pearson and Spearman (rank) correlation coefficients of all determined parameters with the averaged grade and among themselves, respectively, were computed. The two coefficients were found to be similar throughout all parameters, so we restrict ourselves to the discussion of Pearson coefficients. Note that, for a sample size of 20, all correlation coefficients magnitudes larger than 0.5 were considered significant, which corresponds to $p<0.025$ (in fact, the generally accepted value of $p<0.05$ corresponds to $r>0.45$ ). As a further approach to investigate correlations of measured parameters with disease state, the samples were

TABLE 1 Correlation with Mankin grade of all parameters obtained in this study; correlation coefficients in italics are above the significance level of 0.5 with $p<0.05$

\begin{tabular}{|c|c|c|c|}
\hline Property & Spearman coefficient & Pearson coefficient & Device used \\
\hline$\alpha_{<1 \mathrm{MHz}}$ & 0.17 & 0.26 & FFC \\
\hline$\alpha>1 \mathrm{MHz}$ & 0.35 & 0.41 & FFC \\
\hline$A_{Q \text {-peaks }}$ & -0.66 & -0.66 & FFC \\
\hline$T_{1, \max , 0 \text { bar }}$ & -0.30 & -0.50 & MOUSE \\
\hline $\mathrm{T}_{1, \max , 6 \text { bar }}$ & -0.57 & -0.49 & MOUSE \\
\hline change $T_{1, \max , 0}$ bar,6 bar & 0.36 & 0.33 & MOUSE \\
\hline position $T_{1, \max }$ & -0.58 & -0.59 & MOUSE \\
\hline$T_{1, \max } / T_{1, \min }$ & 0.31 & 0.32 & MOUSE \\
\hline thicknesso bar & -0.48 & -0.58 & MOUSE \\
\hline thickness $_{6}$ bar & -0.55 & -0.63 & MOUSE \\
\hline change thickness 0 bar,6 bar & 0.57 & 0.61 & MOUSE \\
\hline position $T_{1, \max } /$ thicknesso bar & 0.36 & 0.36 & MOUSE \\
\hline signal $_{\text {max }, 0 \text { bar }}$ & -0.31 & -0.41 & MOUSE \\
\hline signal $_{\max , 6 \text { bar }}$ & -0.44 & -0.38 & MOUSE \\
\hline change signal ${ }_{\max , 0 \text { bar,6 bar }}$ & 0.04 & 0.03 & MOUSE \\
\hline area signalo bar $_{1}$ area signal 6 bar & 0.42 & 0.50 & MOUSE \\
\hline$T_{1,11.7 \mathrm{MHz}}$ & -0.10 & -0.20 & FFC \\
\hline$T_{1, \text { low field }}$ & 0.27 & 0.32 & FFC \\
\hline$T_{2, \max , 0 \text { bar }}$ & -0.63 & -0.48 & MOUSE \\
\hline$T_{2, \max , 6 \text { bar }}$ & -0.62 & -0.70 & MOUSE \\
\hline change $T_{2, \max , 0}$ bar, 6 bar & 0.19 & 0.17 & MOUSE \\
\hline$T_{2, \max } / T_{2, \min }$ & 0.18 & 0.05 & MOUSE \\
\hline position $T_{2, \max }$ & -0.34 & -0.36 & MOUSE \\
\hline position $T_{2, \max } /$ thickness 0 bar & 0.09 & 0.14 & MOUSE \\
\hline
\end{tabular}


divided into two groups, 'mild OA' and 'severe OA', by a cutoff value of 4.5, resulting in a set of seven 'mild OA' specimens (average Mankin grade 2.38) and 13 'severe $O A^{\prime}$ ' specimens (average 7.92). The cutoff value of 4.5 was determined by considering pannus and/or surface irregularities, diffuse hypercellularity and slight reduction in safranin-O staining as features of 'mild OA'. A two-set $t$-test statistical analysis was carried out on all these parameters with respect to comparing the groups 'mild OA' and 'severe OA', assuming a confidence interval of 0.95 for identifying the parameters as significantly different. The assumption of equal variances for both distributions has been made. All statistical analyses were carried out with Origin $8.5^{\mathrm{TM}}$ software.

\section{3 | RESULTS}

A total of 24 parameters were defined that could be obtained directly, or by comparison between two experiments with and without mechanical load, from the experimental data. Five of them were derived from relaxometry data and the remainder from analysis of the profiles obtained at 0.27 T. These data are listed in Table 1 along with the device on which they were measured. Note that in some cases it was not possible to extract

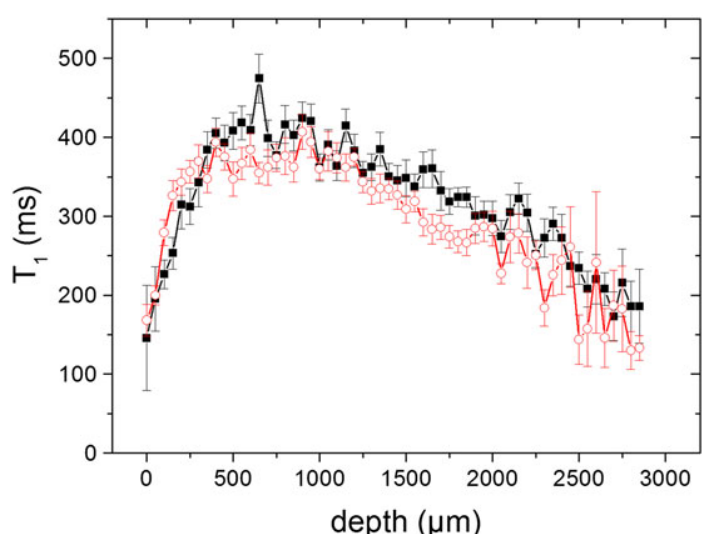

(A)

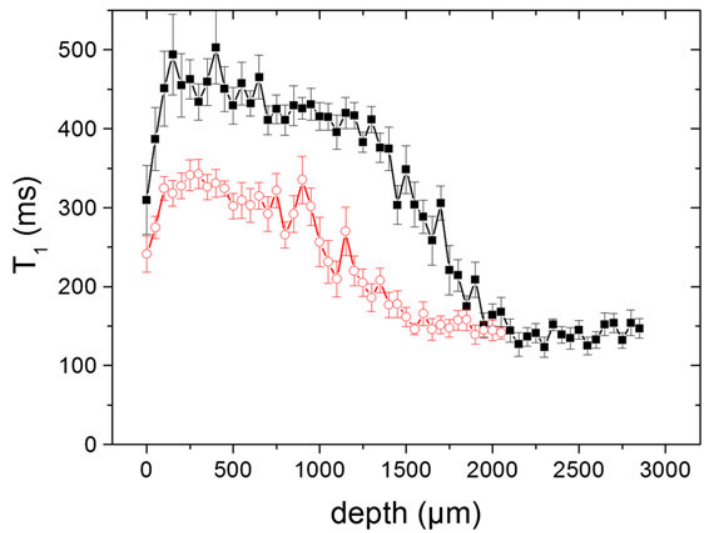

(B)

FIGURE 1 A, $T_{1}$ across healthy (Mankin grade 1) cartilage plug measured at a ${ }^{1} \mathrm{H}$ Larmor frequency of $11.7 \mathrm{MHz}$. $\mathrm{B}$, as in a, but for a sample with severe OA (Mankin grade 11). Filled symbols represent unloaded tissue, open symbols represent loading at $0.6 \mathrm{MPa}$. Depth is counted from the tissue surface

TABLE 2 Results of two-hypothesis t-test of parameters between 'mild OA' and 'severe OA'. Only statistically significant results (confidence level > 95\%) are shown

\begin{tabular}{lcccc} 
Property & Units & 'Mild OA' & 'Severe OA' & Confidence interval (\%) \\
\hline$A_{\text {Q-peaks }}$ & (a.u.) & $1.608 \pm 0.188$ & $0.838 \pm 0.236$ & 95 \\
position $T_{1, \max }$ & (um) & $821 \pm 114$ & $477 \pm 91$ & 95 \\
\hline$T_{1, \text { max,6 bar }}$ & (ms) & $375.1 \pm 8.7$ & $324.0 \pm 11.3$ & 95 \\
thickness 0 bar & (um) & $2417 \pm 145$ & $1868 \pm 168$ & 95 \\
thickness 6 bar & (um) & $1907 \pm 167$ & $1337 \pm 167$ & 95 \\
$T_{2, \text { max,6 bar }}$ & (ms) & $44.8 \pm 1.7$ & $37.7 \pm 1.6$ & 95 \\
\hline
\end{tabular}


all parameters satisfactorily; these points were excluded from the statistical analysis. However, in all cases, the correlation was still above the significance level (i.e., $p<0.05$ ), which will be discussed in this section.

As the result of the two-set $t$-test statistical analysis, it was found that six out of these parameters showed statistically significant differences between the groups defined as 'mild OA' and severe OA': the maximum value inside the tissue for both $T_{1}$ and $T_{2}$ under load of $0.6 \mathrm{MPa}$; the position of the former in the absence of pressure; the sample thickness, measured from the outer sample surface (defined as zero) to the calcified zone surface, with and without load; and the area of the quadrupolar peaks. Their respective averages and correlations are shown in Table 2. No other parameters showed differences that were statistically significant within the confidence interval of 0.95 .

Rather than distinguishing the tissue grade into two relatively arbitrary groups, we further attempted to determine correlations between all measured parameters, including, but not restricted to, the Mankin grade. Since this grade must necessarily be a descriptive parameter and cannot

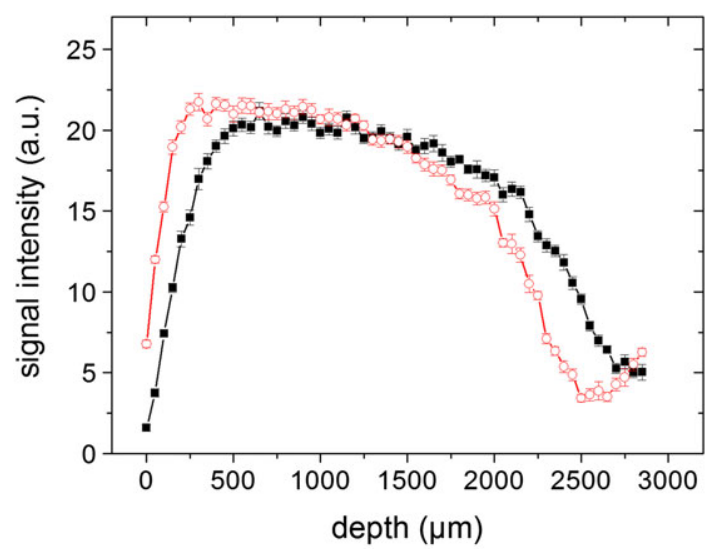

(A)

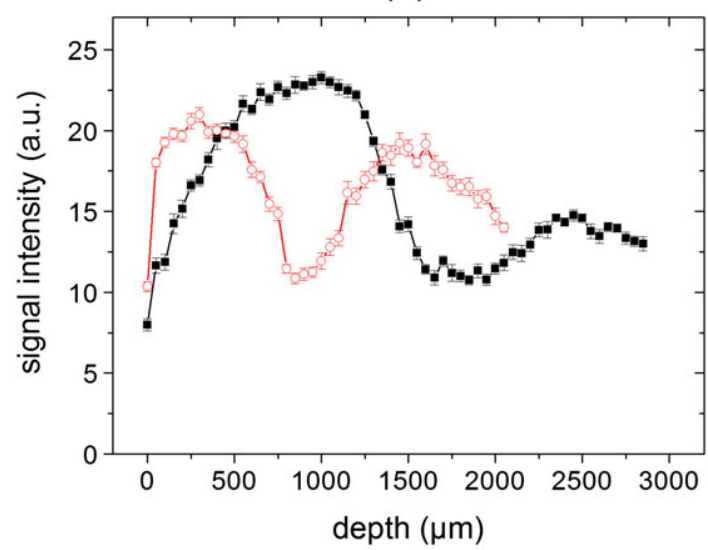

(B)

FIGURE 2 As in Figure 1, but with the signal intensity plotted for the same samples. The signal intensity was obtained from integrating over the first six echoes in the CPMG train

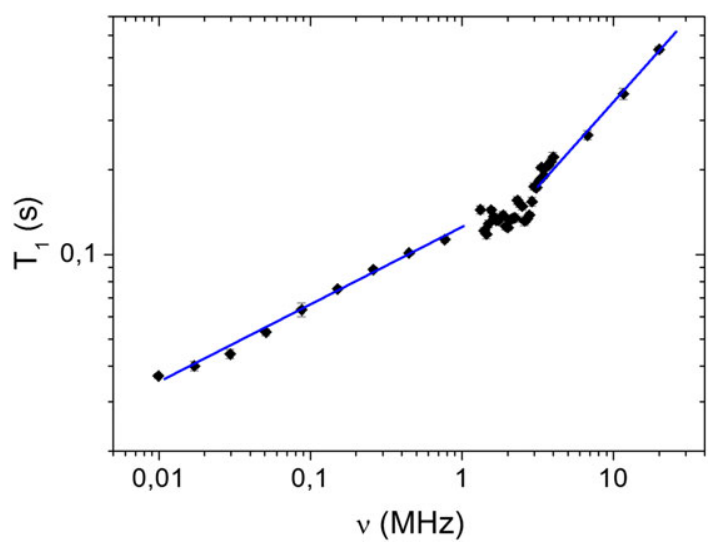

FIGURE 3 Relaxation time as a function of Larmor frequency, $T_{1}(v)$, for a typical cartilage sample, indicating the apparent power laws fitted to the data, and showing the region of quadrupolar dips. Lines are drawn as guides to the eye, symbolizing the two distinct regimes 


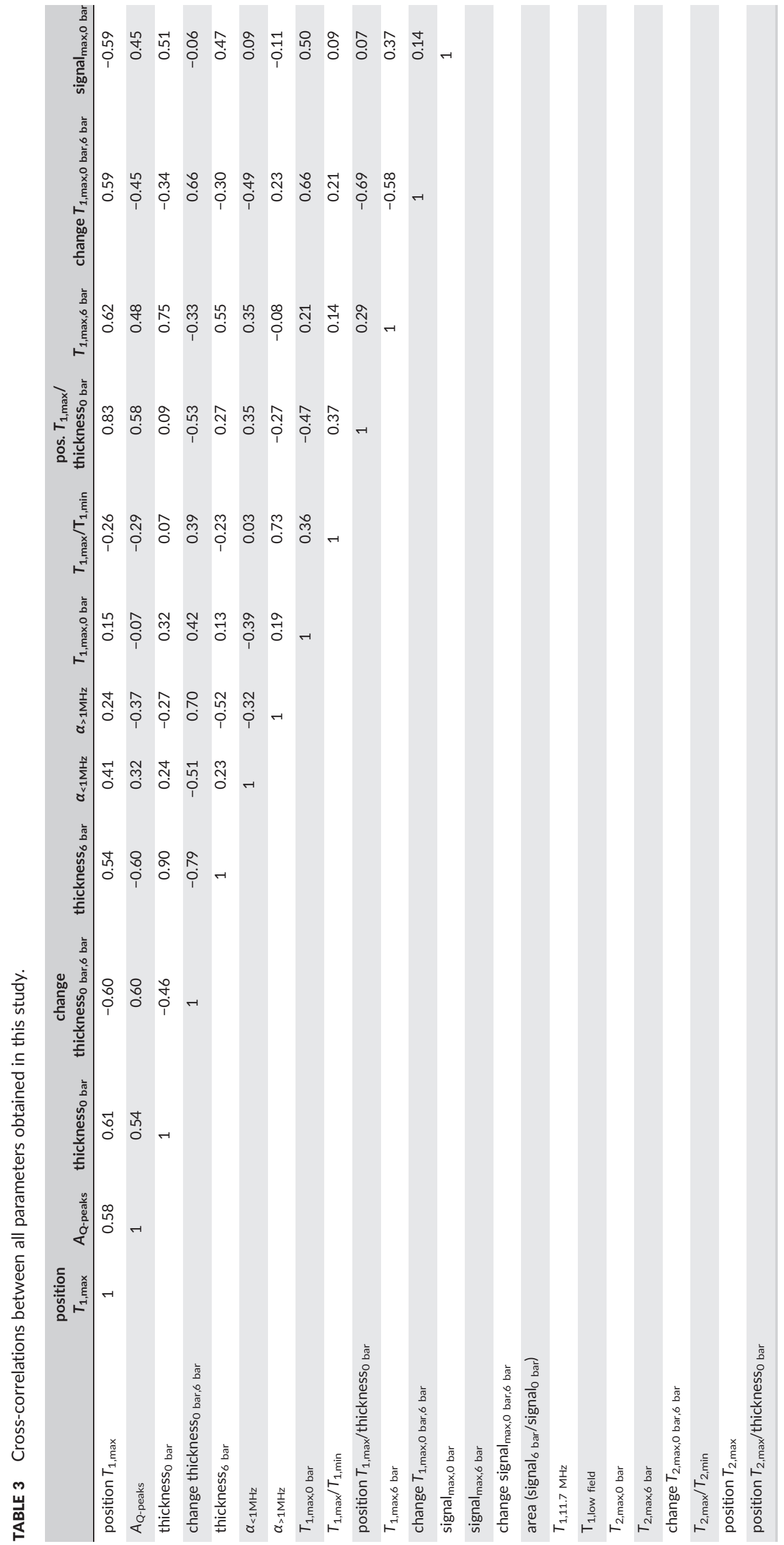




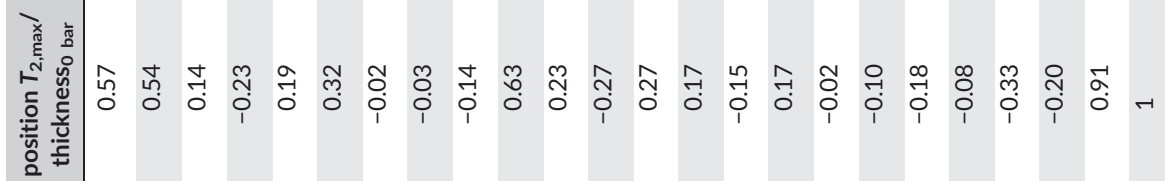

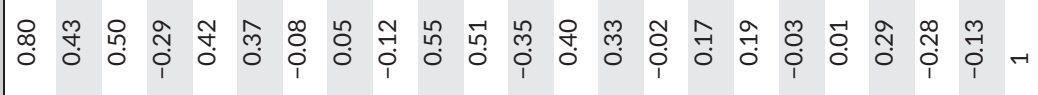
产

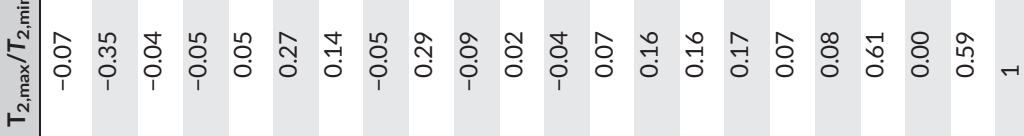

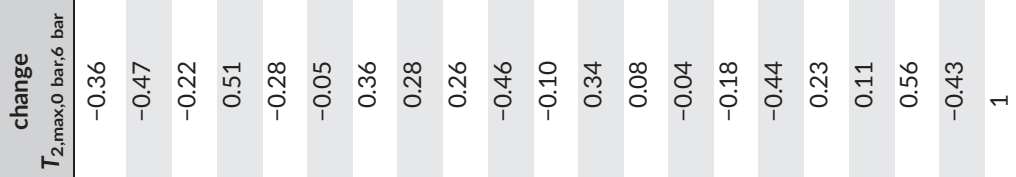

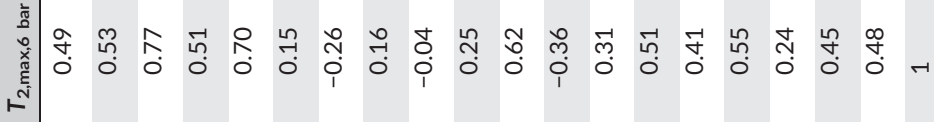

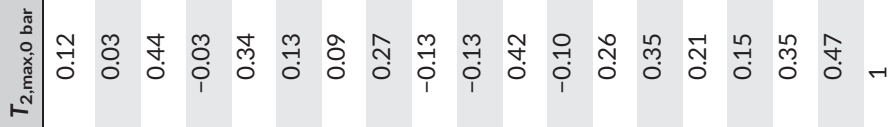

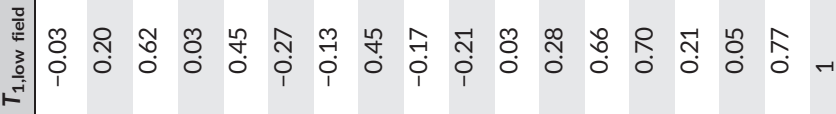

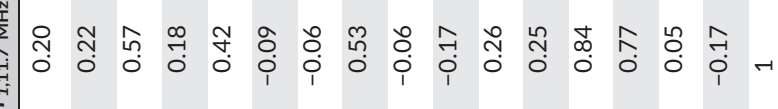

言竞

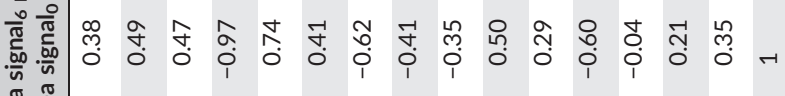

产

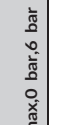

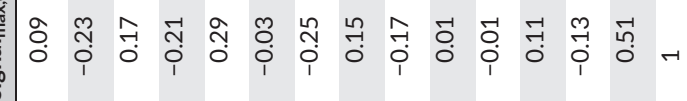

I

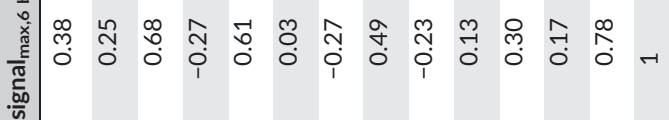

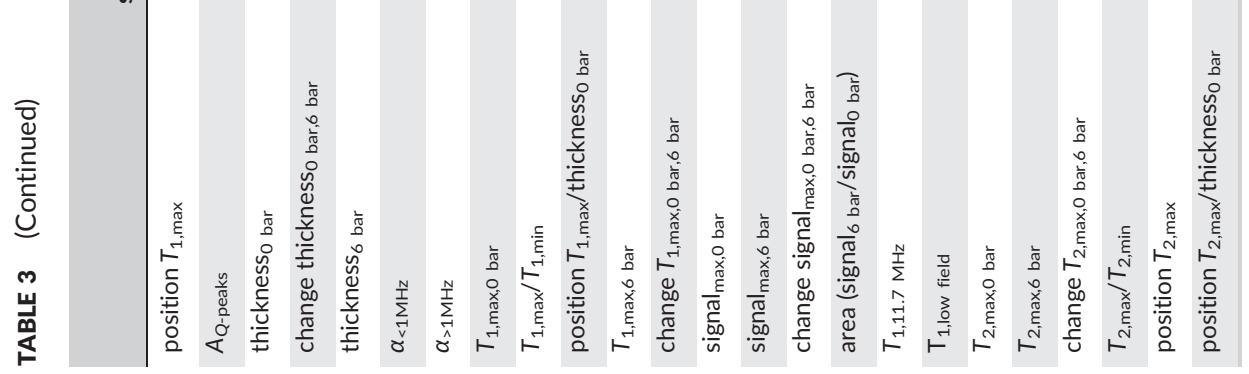


be assumed to behave linearly, the conditions for a regression analysis are only approximately met. Cross-correlation of other parameters is assumed to highlight relations that can be exploited by employing different low-field NMR hardware.

Figures 1 and 2 show typical properties of spatially resolved $T_{1}$ and the corresponding integrated signal intensity, which is approximately equivalent to water content, obtained with NMR-MOUSE at $0.27 \mathrm{~T}\left(11.7 \mathrm{MHz}{ }^{1} \mathrm{H}\right.$ resonance frequency). Note that the experiment yields relaxation times in a thin, planar slice; the curvature of the sample itself will result in some averaging over neighboring regions. This behavior has been thoroughly discussed in Reference 34. For samples lacking sharp edges or discontinuities in distributions, the effect of such curvature is considered minor, and it certainly does not affect significantly the extreme values and the thickness.

Figures $1 \mathrm{~A}$ and $2 \mathrm{~A}$ demonstrate qualitatively the behavior of healthy tissue. While the thickness is reduced by only about $10 \%, T_{1}$ remains apparently unchanged. In contrast, the diseased sample (Mankin Grade 11, Figures 1B, 2B) shows an overall shrinkage of 50\% and clearly distorted

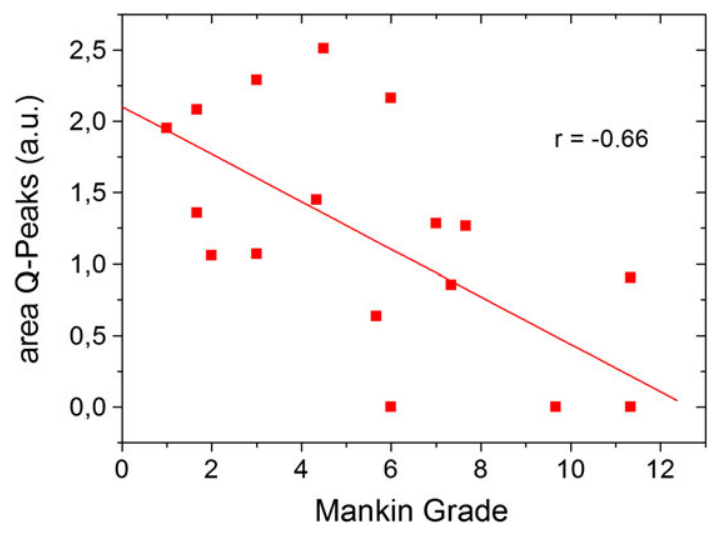

FIGURE 4 Correlation between Mankin grade of cartilage tissues and the area under the quadrupolar relaxation rate peaks (for description see text)

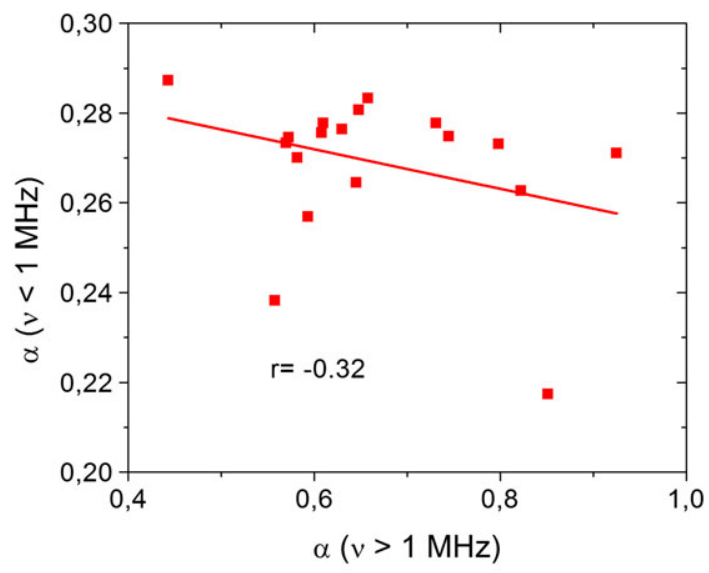

(A)

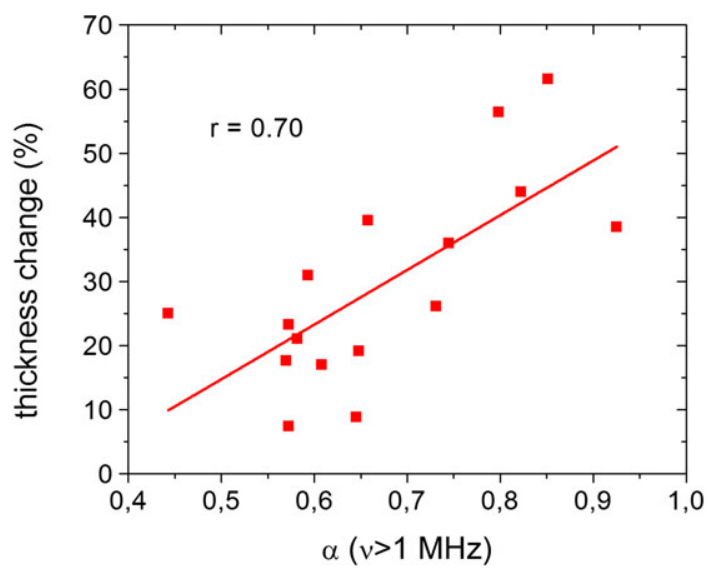

(B)

FIGURE 5 A, Correlation between power-law exponents in the frequency ranges below and above $1 \mathrm{MHz}$, respectively. B, Correlation between thickness change with and without load and power-law exponent in the frequency range between 1 and $20 \mathrm{MHz}$ 
$T_{1}$ profile under pressure: not only is the shape changed, but also the actual value of $T_{1}$ is decreased throughout the tissue, reducing the dynamic range between maximum and minimum approximately from 3 to 2 . This is important in situations when spatial resolution is not available, but the distribution of $T_{1}$ relaxation times can be accessed as an additional measure of sample characterization (see Section 4). The profiles of $T_{2}$ behave accordingly (data not shown), but with larger error bars because of the probably inherent non-exponentiality of the decays due to spatial structural heterogeneity when averaged over the slice. ${ }^{38}$

Figure 3 shows typical dispersion curves for cartilage samples. Common features are an approximate power-law behavior $T_{1} \sim \omega^{\alpha}$ in the lowerfield region, transiting to a steeper dependence up to the highest field accessed in this experiment $\left(0.47 \mathrm{~T}\right.$, corresponding to $20 \mathrm{MHz}{ }^{1} \mathrm{H}$ Larmor frequency), which can tentatively also be approximated by a power law and which is continuing towards the longer $T_{1}$ known from clinical field strengths. In between are the quadrupolar dips, i.e. the effect of additional ${ }^{1} \mathrm{H}^{1}{ }^{14} \mathrm{~N}$ cross-relaxation of mobile water in contact with the amino acids of glycosaminoglycan (GAG) and collagen. The frequencies of these dips depend on the position of the nitrogen nucleus inside the molecule and are close to $2.1 \mathrm{MHz}$ and $2.8 \mathrm{MHz}$, respectively, for amide groups, with a third dip at $0.7 \mathrm{MHz}$ frequently becoming inconspicuous relative to the background relaxation rate. In this study, the areas of the upper two peaks of $R_{1}$ were determined in order to provide a quantifiable parameter. ${ }^{24,39,40}$

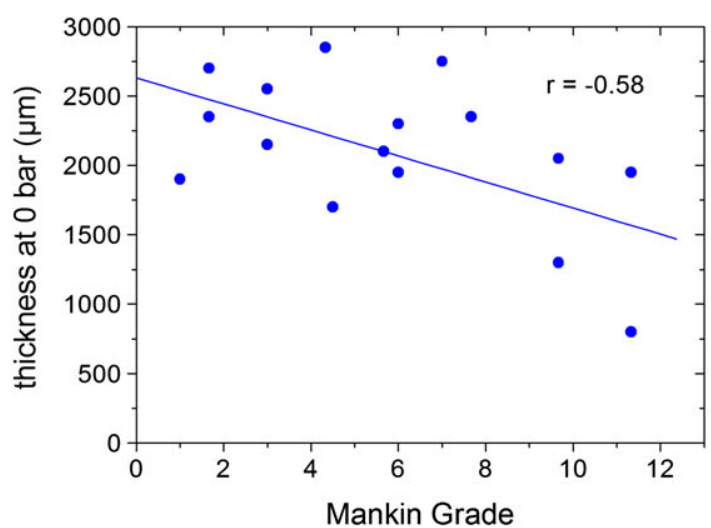

(A)

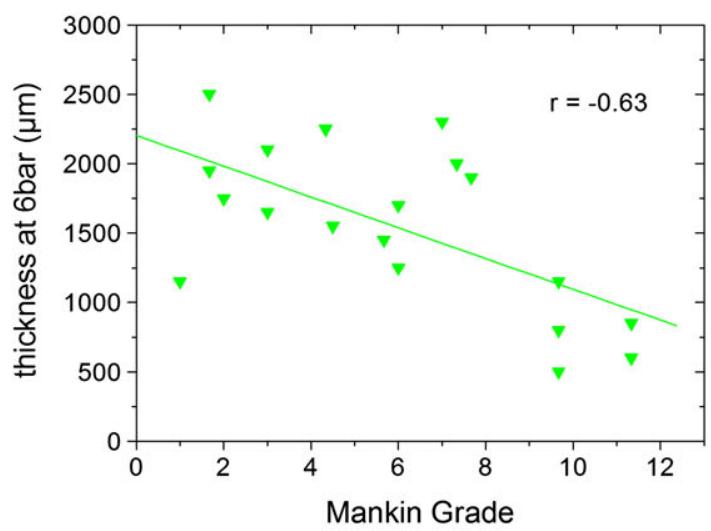

(B)

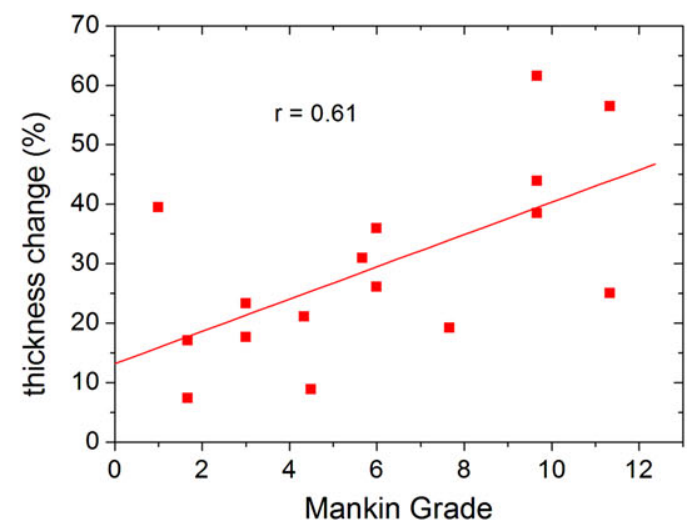

(C)

FIGURE 6 Correlation of thickness without load A, thickness under $0.6 \mathrm{MPa}$ load (B) and relative difference between these two values (C) with Mankin grade. The confidence values for these three values were $p=0.012,0.0022$ and 0.007 , respectively 


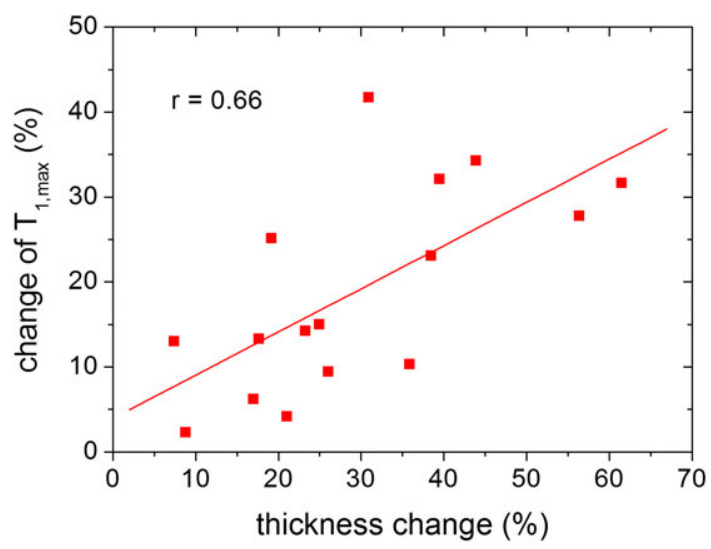

FIGURE 7 Correlation between the change of the maximum $T_{1}$ at $11.7 \mathrm{MHz}$ and the change in thickness, comparing tissue with and without load $(p=0.003)$

Table 3 provides a summary of all correlation coefficients obtained for the investigated samples. In the following, a number of particularly relevant correlations will be highlighted.

First of all, in Figure 4, the dependence of quadrupolar peak area on Mankin grade, as obtained from the relaxometry dispersion measurements, is shown. A negative correlation coefficient of $r=-0.66(p=0.0021)$ is observed; qualitatively, the same finding was reported in Reference 24 in another study of human cartilage samples. The other quantities obtained from the dispersion curves are the power-law exponents themselves, according to the relation $T_{1} \sim \omega^{\alpha}$. Figure 5A demonstrates that no significant correlation was found for the two exponents in the lower- and higher-frequency regions, respectively. While dispersion is essentially unchanged below $1 \mathrm{MHz}$, remaining in a narrow range of $\alpha \approx 0.27 \pm 0.01$, only the slope above $1 \mathrm{MHz}$ correlates with any of the other parameters. The most obvious correlation $(r=0.70, p=0.0012)$ was found with thickness change as a consequence of mechanical load, which, in turn, is inversely proportional to Young's modulus (see Figure 5B).

Tissue thickness is a parameter that is accessible in clinical scanners and therefore has been investigated statistically before. In Figure 6, thickness with and without load, and in particular thickness change between these two conditions, is shown to possess significant correlation with Mankin grade: diseased tissue is, on average, thinner and more compressible. Since thickness change is related to the $E$ modulus, this corresponds to a corroboration of existing reports, which show a strong relationship of modulus and OA. ${ }^{41-43}$

Finally, the change of thickness also correlates with the variation of the maximum value of $T_{1}$ at $11.7 \mathrm{MHz}$, a quantity that is, at least in principle, also accessible in low-resolution systems where the thickness itself cannot be determined. This connection is shown in Figure 7.

\section{4 | DISCUSSION}

The use of low-field and variable-field devices opens up the possibility to obtain parameters that are not commonly accessible in clinical scanners. On the other hand, both low- and high-field modalities have been combined with imaging equipment so that, allowing for certain restrictions in resolution, in vivo studies of cartilage are becoming realistic, despite the fact that no large-scale statistical studies on OA have been reported yet.

\subsection{Relaxation times at fixed field}

The parameters most easily determined are average relaxation times, and indeed positive correlations with OA have been found for $T_{2}$ and $T_{1 \rho}{ }^{2,4-8}$ but not for $T_{1}{ }^{44}$. At the same time, it has been reported that $T_{2}$ is frequently non-exponential even when averaged over rather small volumes of the tissue, and that careful analysis of the different components, as well as their orientation dependence with respect to the axis of the magnetic field, is required to establish and justify such correlations. ${ }^{38} \mathrm{~A}$ superficial explanation for these findings is the enhanced water content known for OA tissue $^{26}$-all other components kept constant, this would increase the amount of 'free' water not affected by any surface, and hence averaging due to fast molecular exchange would lead to longer relaxation times. ${ }^{45}$ The situation must, however, be more complicated, since the depletion of GAG also removes relaxation sinks for water protons, and the rearrangement of water in the weaker OA tissue under compression changes the relative weight of 'free' water.

In this study, no correlation with degeneration state was found for the averaged values, in contrast to other studies such as Reference 6, which was carried out at high field ( $3 \mathrm{~T}$ ), but for the maximum values of both $T_{1}$ and $T_{2}$ under conditions with and without load. These maxima are identified at a spatial resolution of $50 \mu \mathrm{m}$, while one needs to keep in mind that averaging already takes place over the $6 \mathrm{~mm}$ diameter of the cartilage plug. Although this resolution is accessible, and even surpassed, at dedicated microimaging systems in high fields, it is out of reach for clinical scanners. These very same maximum values would therefore not be directly available, but could be extracted by a detailed analysis of the signal decay, in particular applying inverse Laplace transform (ILT) or related techniques to the data. ILT is established for broad distributions of relaxation times, but so far sees only limited use in biomedical studies. Nevertheless, it appears to be a promising tool insofar as it can provide 
the extreme values of a distribution, or its width as an alternative fitting parameter. Corresponding results on biological samples will be discussed in a forthcoming paper.

The maximum of $T_{2}$ typically occurs in the transitional zone (TZ) of cartilage and is connected to the anisotropy of collagen fibrils being highest in this range ${ }^{4}$; in fact, the gradient of relaxation times variation outside this maximum has been used for a precise definition of the boundaries between layers. ${ }^{46-48}$ From earlier studies with bovine articular cartilage, but also from results within this study, it was found that the position of the maximum of $T_{1}$ generally occurs in the vicinity of that of $T_{2}$, while it can be assigned much more easily due to the often multiexponential behavior of $T_{2}$, which is not observed for $T_{1}$. It can thus be assumed that parameters describing the actual maximum value of $T_{1}$ are predominantly related to morphological changes in the TZ, possibly changes of local water content, while variations of its position include changes in the remaining zones as well.

In this study, no correlation of $T_{1, \max }$ with any other parameter, apart from the trivial case of the average $T_{1}$ at $11.7 \mathrm{MHz}$, was observed in the uncompressed sample. This was somewhat unexpected, considering the general increase of relaxation contrast towards lower magnetic field strengths. However, significant correlations were found for $T_{1 \text {,max }}$ under load, such as thickness and thickness change, Mankin grade, maximum signal intensity and also the corresponding value $T_{2 \text {,max. }}$. The combination of these findings indicates that the mobility and content of water in cartilage, in particular within the TZ, is a suitable indicator for OA severity.

The position of $T_{1, \max }$, on the other hand, measured as the distance from the tissue surface, correlates with Mankin grade, Q-dip area, cartilage thickness and thickness change, where the latter two parameters may possibly not be fully independent of each other. However, when normalizing the position within the cartilage, i.e. dividing the value by the thickness measured under the same conditions, the correlation with thickness change still remains but is somewhat weaker ( -0.53 compared with -0.60$)$. This corresponds to the finding that the $T_{1}$ profile across cartilage is not deformed affinely under pressure, but the position of $T_{1, \max }$, in absolute as well as relative scale, moves towards the surface. Note that this observation is at variance with a high-field study, where the weakly pronounced $T_{1}$ variation sees a maximum closer to the top of the cartilage, which then moves inward after the application of mechanical load. ${ }^{49}$ This, however, is not a contradiction, since $T_{1}$ obtained at $7 \mathrm{~T}$ and at $0.27 \mathrm{~T}$ probe entirely different molecular dynamics, as $T_{1}$ is most sensitive to processes on a timescale of the inverse Larmor frequency, i.e. $\omega t=1$. In References 10 and 50, variations of $T_{2}$ and zone thicknesses are reported for canine cartilage tissue, and were found to strongly depend on sub-cartilage tissue and the amount of compression.

\subsection{Shape parameters of cartilage with and without load}

Next to relaxation times come the geometric properties, which are routinely analyzed, at least indirectly, in clinical scans, frequently via the distance between bone surfaces on either side of a joint. ${ }^{51,52}$ Thinning of cartilage during OA is therefore a well-known phenomenon. The negative correlation of tissue thickness with Mankin grade is confirmed in this study. Moreover, the relative thickness change under pressure correlates positively, an observation that is in agreement with earlier studies at high field. ${ }^{53}$ For hardware without sufficient spatial resolution, it is interesting to look at the cross-correlation coefficients with other observables: in particular, these are the parameters derived from variable-field measurements such as power-law and Q-dip area (see below), but also $T_{1, \max }$ at $0.27 \mathrm{~T}$ and its change. It appears intuitively obvious to assume that 'softer' tissue, which contracts more under load, will lead to a larger change in water content, hereby also affecting the distribution of $T_{1}$ and its maximum value. It is also relevant to mention that a weak correlation with the average $T_{1}$ was only found for the thickness without load, not with load, or any other directly disease-related quantities.

\section{3 | Relaxation times at variable field}

Finally, the variable-field study provides parameters not accessible by most conventional instruments. The correlation of quadrupolar dip are, or peak area, with Mankin grade was already mentioned. The origin of these dips is the additional relaxation rate attributed to ${ }^{1} \mathrm{H}$ nuclei in the vicinity of the quadrupolar ${ }^{14} \mathrm{~N}$ nuclei. If the Zeeman energy of the ${ }^{1} \mathrm{H}$ is equal to one of the three quadrupolar energies of ${ }^{14} \mathrm{~N}$, ${ }^{54-56}$ magnetization can be transferred, and will always 'flow' towards the pool of ${ }^{14} \mathrm{~N}$, which equilibrates within a much shorter time; the result is a shortened $T_{1}$ of ${ }^{1} \mathrm{H}$.

These energy levels are defined as

$$
\Omega_{ \pm}=\frac{K}{\hbar}(3 \pm \eta), \quad \Omega_{0}=\frac{2 K}{\hbar} \eta
$$

with $K=\frac{e^{2} q Q}{4}$, where $q$ is the electric field gradient along the $z$ axis of the principal axes system, $Q$ is the quadrupolar moment and $\eta$ is the asymmetry parameter, of the order 0.4 for amide nitrogens. In fact, all amide nitrogens-with a natural abundance of close to $100 \%$-have very similar atomic environment, and thus also nearly identical transition frequencies. The sum of amino acids in GAG and collagen, respectively, gives rise to a particular pattern, but in most biological tissue these dips are in the vicinity of ${ }^{1} \mathrm{H}$ Larmor frequencies of $2.8 \mathrm{MHz}, 2.1 \mathrm{MHz}$ and $0.7 \mathrm{MHz}$, and show only weak temperature dependence. ${ }^{54}$

The prominence of these features in cartilage is related to the concentration of GAG and collagen on the one hand; i.e., GAG depletion as a consequence of $\mathrm{OA}$ is expected to have a negative effect on the area of the dips, which was indeed observed. ${ }^{24}$ In Reference 57 it was shown by using selective enzymes that both the GAG and collagen nitrogens contribute to the dips. On the other hand, the mobilities of the ${ }^{14} \mathrm{~N}-$ 
containing species and of the water molecules also affect the presence of the features: if the interaction is completely averaged out due to rapid tumbling of the molecule with the ${ }^{14} \mathrm{~N}$ attached, as for single amino acids in solution, no effect will be seen. In fact, the enhanced mobility of proteins due to increased water concentration is a second, important contribution to the suppression of the quadrupolar dips. ${ }^{57}$ Generally, both GAG depletion and increasing water concentration are signs of advanced $\mathrm{OA},{ }^{58,59}$ so the two effects combined can explain the negative correlation between quadrupolar dip area and Mankin grade found in this study and in Reference 24.

In contrast to the quadrupolar cross-relaxation, discussion of the overall shape of the $T_{1}$ dispersion, i.e. its dependence on Larmor frequency, must currently remain empirical. While the theory of $T_{1}$ dispersion is often well understood for single-component systems, such as polymer melts or water in contact with interfaces, the complexity of biological tissue prohibits a thorough description. Despite this, the dependence of $T_{1}$ on magnetic field strength, particularly at higher fields where conventional MRI studies take place, has been reported before, and databases have been compiled that allow a better comparison of images obtained at different magnetic field strengths. ${ }^{60}$ Attempts have been made to explain dispersion in various types of tissue in a semi-phenomenological way. ${ }^{61}$ With cartilage being a rather simple system, it can be generally assumed that the dynamics of water interacting with surfaces such as GAG and collagen is mostly responsible for the observed dispersion, although magnetization transfer and therefore influence of the dynamics of the protons of the solid components will certainly be another source, as has been proven for hydrated proteins in the absence of excess water. ${ }^{56,62-64}$

In this study, signal decays were analyzed by monoexponential fitting functions; i.e., the obtained relaxation times represent averages over the total cartilage volume. The dispersion data could be fitted phenomenologically to two different power-law relations $T_{1} \sim \omega^{\alpha}$, with a more pronounced frequency dependence in the upper region above $1 \mathrm{MHz}$. The exponents $\alpha$ were taken as fitting parameters, which are not statistically significantly correlated to each other: i.e., the frequency behavior below $1 \mathrm{MHz}$ was found to be almost identical for all samples; in particular, it does not correlate with any other relaxation figure. The exponent above $1 \mathrm{MHz}$, on the other hand, shows correlations, which is not entirely surprising since this range covers the frequency where the MOUSE measurements were carried out, although a correlation with the actual value of $T_{1}$ does not exist. The dependence on Mankin grade remains below the significance level. The observed relation with thickness change and signal amplitude change, both being quantities related to water content, indicate that the latter plays an important role, although in a somewhat counterintuitive fashion: a higher water content, just as well as an increase of mobility of the restricting medium, would generally increase the average of $T_{1}$ in the full frequency range. This has been found in enzymatically degraded hydrated collagen and GAG, respectively, ${ }^{34}$ but is obviously not seen in actual cartilage with OA, suggesting that the origin of $T_{1}$ dispersion in this tissue is more complicated. At this stage, a detailed explanation cannot be given; we note, however, that the slope in the upper frequency region is a valuable parameter since, it can be obtained from measuring $T_{1}$ at two different field strengths about $20 \mathrm{MHz}$, an approach that is potentially feasible for a number of scanners with limited hardware modification. However, the full range of $T_{1}$ dispersion up to clinically relevant fields needs yet to be investigated in order to arrive at a suitable model for elucidating the actual processes acting on a molecular level, including the known observation of $T_{1}$ converging at high fields irrespective of OA status.

The behavior in the frequency range between $10 \mathrm{kHz}$ and $1 \mathrm{MHz}$ is actually related to the absence of any correlations found for $T_{1}$ at $10 \mathrm{kHz}$, the lowest frequency that could be used in this work. In the limit of zero frequency, $T_{1}, T_{2}$ and $T_{1 \rho}$ become identical, and the same correlations known for $T_{2}$ and $T_{1 p}$ could be expected for $T_{1}$ as well. However, such a similarity is not found in this study; it can therefore be tentatively concluded that the origin of correlations of $T_{2}$ and $T_{1 \rho}$ with OA must be sought in very slow, i.e. below $10 \mathrm{kHz}$, dynamic processes of the water molecules in interaction with their environment, a finding that appears to be supported by studies employing $T_{1 \rho}$ at different locking field strengths, where larger deviations were found towards lower locking frequencies. ${ }^{33}$ Note that typical locking frequencies of MRI equipment are even one or two magnitudes below the lower limit of the field-cycling device used in this work.

\section{5 | CONCLUSIONS}

Low-field and variable-field NMR were combined, for the first time, in a study of human articular cartilage with varying degree of OA. Correlations were found for the area of the quadrupolar peaks in the relaxation profile, as well as the magnitude of the ${ }^{1} \mathrm{H}$ dispersion in the frequency range between 1 and $20 \mathrm{MHz}$, expressed by a power-law exponent. Only maximum values of relaxation times within the tissue were found to correlate with $\mathrm{OA}$, not average values, pointing to the need for developing improved analysis algorithms for non-exponential relaxation decays. In general, applying unidirectional load of 0.6 MPa onto cartilage plugs enhanced the correlation of observed quantities; in particular, the corresponding change with/without load in $T_{1}$ relaxation time properties is identified as a promising indicator for OA severity. The findings in this study, and the rather large width of relaxation times values within cartilage tissue, suggest the possibility to obtain even higher contrast for imaging systems operating at variable, ${ }^{28}$ low $^{65}$ or ultralow magnetic fields. ${ }^{66,67}$

\section{ACKNOWLEDGEMENTS}

Part of this work was supported by the EU Horizon 2020 collaborative project IDentIFY (project number 668119). ER gratefully acknowledges Carl Zeiss Stiftung for the scholarship to pursue his PhD research. MTN is indebted to Jane and Aatos Erkko Foundation, Finland for financial support. SS wishes to thank Professor Yang Xia for continuous valuable discussions. The assistance of Maarit Valkealahti in acquiring osteochondral specimens is gratefully acknowledged. 


\section{REFERENCES}

1. Eckstein F, Charles HC, Buck RJ, et al. Accuracy and precision of quantitative assessment of cartilage morphology by magnetic resonance imaging at 3.0T. Arthritis Rheum. 2005;52:3132-3136.

2. Xia Y, Farquhar T, Burton-Wurster N, Ray E, Jelinski LW. Diffusion and relaxation mapping of cartilage-bone plugs and excised disks using microscopic magnetic resonance imaging. Magn Reson Med. 1994;31:273-282.

3. Nieminen MT, Rieppo J, Töyräs J, et al. $T_{2}$ relaxation reveals spatial collagen architecture in articular cartilage: A comparative quantitative MRI and polarized light microscopic study. Magn Reson Med. 2001;46:487-493.

4. Xia Y, Moody JB, Alhadlaq H. Orientational dependence of $T_{2}$ relaxation in articular cartilage: A microscopic MRI ( $\mu$ MRI) study. Magn Reson Med. 2002;48:460-469.

5. David-Vaudey E, Ghosh S, Ries M, Majumdar S. T2 relaxation time measurements in osteoarthritis. Magn Reson Med. 2004;22:673-682.

6. Li X, Pai A, Blumenkrantz G, et al. Spatial distribution and relationship of $T_{1}$ and $T_{2}$ relaxation times in knee cartilage with osteoarthritis. Magn Reson Med. 2009;61:1310-1318.

7. Souza RB, Feeley BT, Zarins ZA, Link TM, Li X, Majumdar S. T1rho MRI relaxation in knee OA subjects with varying sizes of cartilage lesions. Knee. 2013;20:113-119.

8. Prasad AP, Nardo L, Schooler J, Joseph GB, Link TM. $T_{1 \rho}$ and $T_{2}$ relaxation times predict progression of knee osteoarthritis. Osteoarthr Cartil. 2013;21:69-76

9. Deng X, Farley M, Nieminen MT, Gray M, Burstein D. Diffusion tensor imaging of native and degenerated human articular cartilage. Magn Reson Imaging. 2007;25:168-171.

10. Lee JH, Badar F, Kahn D, Matyas J, Qu X, Xia Y. Loading-induced changes on topographical distributions of the zonal properties of osteoarthritic tibial cartilage-A study by magnetic resonance imaging at microscopic resolution. J Biomech. 2015;48:3625-3633.

11. Mayerhoefer ME, Welsch GH, Mamisch TC, et al. The in vivo effects of unloading and compression on T1-Gd (dGEMRIC) relaxation times in healthy articular knee cartilage at 3.0 Tesla. Eur Radiol. 2010;20:443-449.

12. Subburaj K, Souza RB, Stehling C, et al. Association of MR relaxation and cartilage deformation in knee osteoarthritis. J Orthop Res. 2012;30:919-926.

13. Xia Y, Momot K (Eds). Biophysics and Biochemistry of Cartilage by NMR and MRI. London: Royal Society of Chemistry; 2016.

14. Dahabreh IJ, Hadar N, Chung M. Emerging magnetic resonance imaging technologies for musculoskeletal imaging under loading stress: Scope of the literature. Ann Intern Med. 2011;155:616-624.

15. Herberhold C, Faber S, Stammberger T, et al. In situ measurement of articular cartilage deformation in intact femoropatellar joints under static loading. J Biomech. 1999;32:1287-1295.

16. Gold GE, Besier TF, Draper CE, Asakawa DS, Delp SL, Beaupré GS. Weight-bearing MRI of patellofemoral joint cartilage contact area. J Magn Reson Imaging. 2004;20:526-530.

17. Besier TF, Draper CE, Gold GE, Beaupré GS, Delp SL. Patellofemoral joint contact area increases with knee flexion and weight-bearing. J Orthop Res. 2005;23:345-350.

18. Adams ME, Li DKB, McConkey JP, et al. Evaluation of cartilage lesions by magnetic resonance imaging at $0.15 \mathrm{~T}-$ comparison with anatomy and concordance with arthroscopy. J Rheumatol. 1991;18:1573-1580.

19. Yoshioka $\mathrm{H}$, Ito $\mathrm{S}$, Handa $\mathrm{S}$, et al. Low-field compact magnetic resonance imaging system for the hand and wrist in rheumatoid arthritis. J Magn Reson Imaging. 2006;23:370-376.

20. Qazi AA, Folkesson J, Pettersen PC, Karsdal MA, Christiansen C, Dam EB. Separation of healthy and early osteoarthritis by automatic quantification of cartilage homogeneity. Osteoarthr Cartil. 2007;15:199-1206.

21. Link TM, Stahl R, Woertler K. Cartilage imaging: Motivation, techniques, current and future significance. Eur Radiol. 2007;17:1135-1146.

22. Ostendorf B, Edelmann E, Kellner H, Scherer A. Low-field magnetic resonance imaging for rheumatoid arthritis. Z Rheumatol. 2010;69:79-86.

23. Kimmich R, Anoardo E. Field-cycling NMR relaxometry. Prog Nucl Magn Reson Spectrosc. 2004;44:257-320.

24. Broche LM, Ashcroft GP, Lurie DJ. Detection of osteoarthritis in knee and hip joints by fast field-cycling NMR. Magn Reson Med. 2012;68:358-362.

25. Rössler E, Mattea C, Mollova A, Stapf S. Low-field one-dimensional and direction-dependent relaxation imaging of bovine articular cartilage. J Magn Reson. 2011;213:112-118.

26. Berberat JE, Nissi MJ, Jurvelin JS, Nieminen MT. Assessment of interstitial water content of articular cartilage with $T_{1}$ relaxation. Magn Reson Imaging. 2009;27:727-732.

27. Blümich B, Perlo J, Casanova F. Mobile single-sided NMR. Prog Nucl Magn Reson Spectrosc. 2008;52:197-269.

28. Lurie DJ, Aime S, Baronic S, et al. Fast field-cycling MRI. C R Phys. 2010;11:136-148.

29. Pine KJ, Goldie F, Lurie DJ. In vivo field-cycling relaxometry using an insert coil for magnetic field offset. Magn Reson Med. 2014;72:1492-1497.

30. Ross PJ, Broche LM, Lurie DJ. Rapid field-cycling MRI using fast spin echo. Magn Reson Med. 2015;73:1120-1124.

31. Alford JK, Rutt BK, Scholl TJ, Handler WB, Chronik BA. Delta relaxation enhanced MR: Improving activation-enhanced specificity of molecular probes through $R_{1}$ dispersion imaging. Magn Reson Med. 2009;61:796-802.

32. Harris CT, Handler WB, Araya Y, et al. Development and optimization of hardware for delta relaxation enhanced MRI. Magn Reson Med. 2014;72:11821189.

33. Rautiainen J, Nissi MJ, Salo E-N, et al. Multiparametric MRI assessment of articular cartilage degradation: Correlation with quantitative histology and mechanical properties. Magn Reson Med. 2015;74:249-259.

34. Rössler E, Mattea C, Stapf S. Feasibility of high-resolution one-dimensional relaxation imaging at low magnetic field using a single-sided NMR scanner applied to articular cartilage. J Magn Reson. 2015;251:43-51.

35. Mankin HJ, Dorfman H, Lippiell L, Zarins A. Biochemical and metabolic abnormalities in articular cartilage from osteo-arthritic human hips. 2. Correlation of morphology with biochemical and metabolic data. J Bone Joint Surg A. 1971;53:523-537. 
36. Pritzker KPH, Gay S, Jimenez SA, et al. Osteoarthritis cartilage histopathology: Grading and staging. Osteoarthr Cartil. 2006;14:13-29.

37. Pauli C, Whiteside R, Heras FL, et al. Comparison of cartilage histopathology assessment systems on human knee joints at all stages of osteoarthritis development. Osteoarthr Cartil. 2012;20:476-485.

38. Zheng SQ, Xia Y. Multi-component of $T_{2}$ relaxation in ex vivo cartilage and tendon. J Magn Reson. 2009;198:188-196.

39. Jiao X, Bryant RG. Noninvasive measurement of protein concentration. Magn Reson Med. 1996;35:159-161.

40. Broche LM, Ismail SR, Booth NA, Lurie DJ. Measurement of fibrin concentration by fast field-cycling NMR. Magn Reson Med. 2012;67:14531457.

41. Setton LA, Elliott DM, Mow VC. Altered mechanics of cartilage with osteoarthritis: Human osteoarthritis and an experimental model of joint degeneration. Osteoarthr Cartil. 1999;7:2-14.

42. Alhadlaq HA, Xia Y, Moody JB, Matyas JR. Detecting structural changes in early experimental osteoarthritis of tibial cartilage by microscopic magnetic resonance imaging and polarised light microscopy. Ann Rheum Dis. 2004;63:709-717.

43. Knecht S, Vanwanseele B, Stüssi E. A review on the mechanical quality of articular cartilage-limplications for the diagnosis of osteoarthritis. Clin Biomech. 2006;21:999-1012.

44. Nissi MJ, Töyräs J, Laasanen MS, et al. Proteoglycan and collagen sensitive MRI evaluation of normal and degenerated articular cartilage. J Orthop Res. 2004;22:557-564.

45. Zimmerman JR, Brittin WE. Nuclear magnetic resonance studies in multiple phase systems: Lifetime of a water molecule in an adsorbing phase on silica gel. J Phys Chem. 1957;61:1328-1333.

46. Xia Y, Moody JB, Burton-Wurster N, Lust G. Quantitative in situ correlation between microscopic MRI and polarized light microscopy studies of articular cartilage. Osteoarthr Cartil. 2001;9:393-406.

47. Kurkijärvi JE, Nissi MJ, Rieppo J, et al. The zonal architecture of human articular cartilage described by $\mathrm{T}_{2}$ relaxation time in presence of Gd-DTPA ${ }^{2-}$. Magn Reson Imaging. 2008;26:602-607.

48. Lee JH, Xia Y. Quantitative zonal differentiation of articular cartilage by microscopic magnetic resonance imaging, polarized light microscopy, and Fourier-transform infrared imaging. Microsc Res Tech. 2013;76:625-632.

49. Xia Y, Wang N, Lee J, Badar F. Strain dependent $T_{1}$ relaxation profiles in articular cartilage by MRI at microscopic resolutions. Magn Reson Med. 2011;65:1733-1737.

50. Lee JH, Badar F, Kahn D, et al. Topographical variations of the strain-dependent zonal properties of tibial articular cartilage by microscopic MRI. Connect Tissue Res. 2014;55:205-216.

51. Cohen ZA, McCarthy DM, Kwak SD, et al. Knee cartilage topography, thickness, and contact areas from MRI: In-vitro calibration and in-vivo measurements. Osteoarthr Cartil. 1999;7:95-109.

52. Burgkart R, Glaser C, Hyhlik-Dürr A, Englmeier K-H, Reiser M, Eckstein F. Magnetic resonance imaging-based assessment of cartilage loss in severe osteoarthritis. Arthritis Rheum. 2001;44:2072-2077.

53. Raynauld J-P, Martel-Pelletier J, Berthiaume M-J, et al. Quantitative magnetic resonance imaging evaluation of knee osteoarthritis progression over two years and correlation with clinical symptoms and radiologic changes. Arthritis Rheum. 2004;50:476-387.

54. Sunde EP, Halle B. Mechanism of ${ }^{1} \mathrm{H}^{-14} \mathrm{~N}$ cross-relaxation in immobilized proteins. J Magn Reson. 2010;203:257-273.

55. Winter F, Kimmich R. 14N1H and 2H1H cross-relaxation in hydrated proteins. Biophys J. 1985;48:331-335.

56. Kimmich R, Winter F, Nusser W, Spohn KH. Interactions and fluctuations deduced from proton field-cycling relaxation spectroscopy of polypeptides, DNA, muscles and algae. J Magn Reson. 1986;68:263-282.

57. Rössler E, Mattea C, Stapf S. NMRD investigations of enzymatically degraded bovine articular cartilage. Magn Reson Med. 2015;73:2005-2014.

58. Bashir A, Gray ML, Hartke J, Burstein D. Nondestructive imaging of human cartilage glycosaminoglycan concentration by MRI. Magn Reson Med. 1999;41:857-865.

59. Venn M, Maroudas A. Chemical composition and swelling of normal and osteoarthritic femoral-head cartilage. 1. Chemical composition. Ann Rheum Dis. 1977;36:121-129.

60. Bottomley PA, Foster TH, Argersinger RE, Pfeifer LM. A review of normal tissue hydrogen NMR relaxation times and relaxation mechanisms from 1-100 $\mathrm{MHz}-$ Ddependence on tissue type, NMR frequency, temperature, species, excision, and age. Med Phys. 1984;11:425-448.

61. Diakova G, Korb JP, Bryant RG. The magnetic field dependence of water $T_{1}$ in tissues. Magn Reson Med. 2012;68:272-277.

62. Korb JP, Bryant RG. The physical basis for the magnetic field dependence of proton spin-lattice relaxation rates in proteins. $J$ Chem Phys. 2001;115:10964-10974.

63. Daszkiewicz OK, Hennel JW, Szczepkowski TW, Lubas B. Proton magnetic relaxation and protein hydration. Nature. 1963;200:1006-1007.

64. Winter F, Kimmich R. Spin lattice relaxation of dipole nuclei $(I=1 / 2)$ coupled to quadrupole nuclei $(S=1)$. Mol Phys. 1982;45:33-49.

65. Cooley CZ, Stockmann JP, Armstrong BD, et al. Two-dimensional imaging in a lightweight portable MRI scanner without gradient coils. Magn Reson Med. 2015;73:872-883.

66. Inglis B, Buckenmaier K, SanGiorgio P, Pedersen AF, Nichols MA, Clarke J. MRI of the human brain at 130 microtesla. Proc Natl Acad Sci U S A. 2013;110:19194-19201.

67. Espy M, Matlashov A, Volegov P. SQUID-detected ultra-low field MRI. J Magn Reson. 2013;229:127-141.

How to cite this article: Rössler E, Mattea C, Saarakkala S, et al. Correlations of low-field NMR and variable-field NMR parameters with osteoarthritis in human articular cartilage under load. NMR in Biomedicine. 2017:30:e3738. https://doi.org/10.1002/nbm.3738 